\title{
Partial reinforcement delayed extinction effect after regularly alternating reward training
}

\author{
MASATO ISHIDA \\ Osaka University of Education, Osaka, Japan
}

\begin{abstract}
When extinction is delayed very long, the superior resistance to extinction of the random schedule group relative to the alternating schedule group disappears (partial reinforcement delayed extinction effect, PRDE). Two experiments assessed the effects of reinforcement/nonreinforcement on Trial 1 on the PRDE. Following extended partial reinforcement acquisition training in a runway, rats received extinction training after a short (1-day) or long (23-day) retention interval. The schedules used in Experiment 1 were: a single-alternation (SA) schedule beginning each day with a rewarded (r) trial, for Group r-SA; an SA schedule beginning with a nonrewarded (n) trial, for Group n-SA; and a random (Rd) schedule, for Group Rd. The schedules and group names used in Experiment 2 were r-SA, Rd, and r-Rd. The results were that (1) rats given r-SA schedules yielded considerable resistance under delayed extinction, (2) those given Rd and $r-R d$ schedules showed a decline in resistance to extinction over a long retention interval, (3) those given the n-SA schedule showed relatively low resistance at both retention intervals, although retention deficit was not greater than in the case of the Rd schedule, and thus, (4) the PRDE was found in both experiments, although only weakly in Experiment 1 . The results indicated that a regularly alternating reward pattern was a more important determinant than was type of reward on Trial 1 for the PRDE. The PRDE due to differential retention deficits among schedules is discussed on the basis of dual-process associative sequential mechanisms and cognitive rule-encoding mechanisms.
\end{abstract}

Resistance to extinction following various reinforcement schedules has been described appropriately in terms of sequential variables derived from the stimulus-aftereffect hypothesis (Capaldi, 1967). This theory stresses the associative conditioning and generalization of sequentially occurring stimulus aftereffects. Numerous alleyway studies have assessed resistance to extinction on the basis of the performance shown in extinction given immediately after the end of acquisition (immediate extinction). Usually, the interval between the end of acquisition and the start of extinction (A-E interval) was less than $24 \mathrm{~h}$.

However, when the beginning of the extinction trials has been delayed (delayed extinction), different aspects have been found. Ishida (1981) trained in a runway three groups of rats on different reinforcement schedules: single alternation (SA), random (Rd) alternation, and continuous reinforcement (CR). Half of the rats received extinction at a short (1-day) retention interval, and the other half received extinction at a long (23-day) retention interval. Under immediate extinction, resistance to extinction was greatest in the Rd group, less in the SA group, and least in the CR group. However, under delayed extinction, this relationship was partly reversed; that is, the SA revealed higher resistance than the $R d$, and the $C R$ revealed still less resistance than the two partial reinforcement groups. Subsequent study using a double-alternation (DA) schedule (Ishida, 1983) found some similar and

The author's mailing address is: Department of Psychology, Osaka University of Education, Tennoji-ku, Osaka 543, Japan. some different results. Under immediate extinction, the Rd group was more resistant than the DA group. But under delayed extinction, the two groups did not differ from each other. The phenomenon common to both studies was that superior resistance to extinction of the Rd schedule group relative to that of the alternating schedule group disappeared when extinction was delayed. We wish to call this phenomenon the partial reinforcement delayed extinction effect (PRDE).

The results obtained under immediate extinction were consistent with those of previous studies (e.g., Campbell, Knouse, \& Wroten, 1970; Capaldi, 1958; Capaldi \& Minkoff, 1967; Habu \& Ono, 1969; Tyler, Wortz, \& Bitterman, 1953) and were predictable from the sequential variables (Capaldi, 1967). However, the rest of the results, including the PRDE, cannot be explained by these variables. The PRDE is attributable to the fact that the retention deficit as measured by resistance to extinction is less in the rats given the alternating schedule than in those given the random schedule. It seems that learning the reinforcement pattern influences the magnitude of resistance to extinction and prevents the retention deficit in the alternating schedule groups.

In previous studies using single alternation (Ishida, 1981) and double alternation (Ishida, 1983), the intitial trial of each acquisition day was always rewarded. Unpublished data recently collected in our laboratory offer some additional information. The fact that with the SA schedule the daily session during acquisition was always initiated by a reward trial raised the question of whether 
the first trial might be crucial in producing the PRDE. Therefore, an experimental study varying the type of reinforcement on the first trial $\left(T_{1}\right)$ is needed to develop a theory concerning the PRDE.

Experiment 1 was conducted to assess the effects of reinforcement/nonreinforcement on $T_{1}$ on the PRDE employing the SA schedule. All rats received extended acquisition training with very massed trials in order to investigate the relationship between the PRDE and the patterning effect, fast running on the reinforced trials and slow running on the nonreinforced trials. A review of studies reporting the patterning effect shows that the above two conditions may be the important factors in producing an SA patterning effect (e.g., Bloom \& Capaldi, 1961; Capaldi, 1958; Jobe, Mellgren, Feinberg, Littlejohn, \& Rigby, 1977).

\section{EXPERIMENT 1}

\section{Method}

Subjects

The subjects were 48 male rats of the Wistar strain, about 110 days old at the start of the experiment.

\begin{abstract}
Apparatus
The straight alley, which was painted gray and covered with .7$\mathrm{cm}$ clear acrylic glass, was $160 \mathrm{~cm}$ long, $8 \mathrm{~cm}$ wide, and $13 \mathrm{~cm}$ high. Manually operated guillotine doors separated the first $25 \mathrm{~cm}$ and the last $35 \mathrm{~cm}$ of alley into the startbox and goalbox compartments, respectively. Response times were recorded on three electric timers. When the first guillotine door was raised, the first clock started; it stopped when a subject interrupted a photobeam $10 \mathrm{~cm}$ from the first door (start time). The interruption of this first beam also started the second clock. When a second beam, $80 \mathrm{~cm}$ beyond the first, was interrupted, the second clock stopped (runway time) and the third clock started. The third clock stopped (goal time) when the rat broke the beam $25 \mathrm{~cm}$ from the second beam and $20 \mathrm{~cm}$ from the endwall of the goalbox compartment. The food cup, painted gray, was a $6 \times 6 \times 5 \mathrm{~cm}$ cube box and was attached to the endwall.
\end{abstract}

\section{Procedure}

Preliminary training. Eighteen days before the experimental training began, the rats were housed individually and put on a feeding schedule of $17 \mathrm{~g}$ of food per day. Water was always available. Day 5 of this period began pretraining, which lasted until Day 18. During pretraining, all animals were handled daily for $3 \mathrm{~min}$. From Day 9 to Day 18, each rat was allowed to explore the inside of the apparatus for $2 \mathrm{~min}$ a day. No food was given in the apparatus during exploration, and all circuits were turned on to adapt the subjects to the various equipment noises. During pretraining, each rat received two .06-g food pellets in the home cage.

Experimental training. On Day 19, the first day of acquisition training, the subjects were divided randomly into three basic groups of 16 rats each, and assigned to each of three reinforcement schedules ( $r-S A, n-S A$, and Rd). After acquisition training, each basic group was further divided into two groups $(n=8)$, and then given the extinction trials. Thus, six groups of 8 rats each were formed for the present experiment. For half of the rats in each basic group, extinction training was begun on the day after the end of acquisition training; for the other half, extinction training was begun on the 23rd day after the end of acquisition training.

In acquisition training, 12 trials were given daily for 18 days (for a total of 216 trials). Group Rd received $50 \%$ random reinforcement, and the type of reinforcement on $T_{1}$ was randomized across days. The Rd schedule included three different $N$-lengths $\left(N_{1}, N_{2}\right.$, and $N_{3}$ ), which were repeated many times during acquisition. Groups r-SA and n-SA received an SA schedule. However, the former initiated the daily sequence on a rewarded $(R)$ trial and the latter initiated the daily sequence on a nonrewarded $(N)$ trial. During immediate and delayed extinction, $12 \mathrm{~N}$ trials were given daily for 5 days. On $R$ trials, the animals received $20 \mathrm{sec}$ of free access to two .06-g pellets. On $\mathrm{N}$ trials, they were confined to the goalbox for $20 \mathrm{sec}$. The intertrial interval was $20 \mathrm{sec}$. The running order of rats was counterbalanced across subjects. If the rat failed to traverse each alley section within $60 \mathrm{sec}$, it was guided to the goalbox and assigned a time score of $60 \mathrm{sec}$ in each section throughout the experiment.

Daily food, which was a $17-g$ ration minus the amount eaten in the apparatus, was provided $30 \mathrm{~min}$ after the last trial of the day during the acquisition and extinction periods. During an intertrial interval between the last day of acquisition and the first day of extinction, the food schedule was identical to that used during acquisition training.

Results
Three rats died during the experiment and 3 were removed because of illness or procedural error. A total of 42 rats were available for the analyses.

All analyses reported for both Experiment 1 and Experiment 2 are based on the total speed, which was obtained by calculating the reciprocal of the combined start, runway, and goal times, since the results did not vary as a function of alley section.

\section{Acquisition}

An analysis of variance on the mean running speeds for the three basic groups (each $n=14$ ) indicated that there were significant groups $[F(2,39)=8.51, p<.01]$ and days $[F(17,663)=18.54, p<.01]$ effects. The groups $\times$ days interaction was also significant $[F(34,663)=$ $1.90, p<.01]$. An analysis of variance performed on the means of the last day of acquisition (terminal level) showed significant differences among the three groups $[F(2,39)=18.91, p<.01]$. Tukey's posttests indicated that Group $\mathrm{Rd}$ ran more rapidly than either of the remaining groups $(p \mathrm{~s}<.05)$, with no significant difference between the latter two groups. Groups r-SA and n-SA ran rapidly on $R$ trials and slowly on $\mathrm{N}$ trials as acquisition days progressed; Group Rd did not run differentially. On the last day of acquisition, the means of the $R$ trials and the $\mathrm{N}$ trials were, respectively, 0.65 and 0.37 for Group r-SA, 0.65 and 0.42 for Group n-SA, and 1.02 and 0.93 for Group Rd. A $3 \times 2$ factorial analysis of variance indicated that there were significant groups $[F(2,39)=14.31$, $p<.01]$ and type of trial $[F(1,39)=5.36, p<.05]$ effects. The interaction of groups $\times$ type of trial was not significant $(F<1)$. Tukey's posttests indicated that there was a significant difference between the speeds on $R$ and $\mathrm{N}$ trials for Groups r-SA and $\mathrm{n}-\mathrm{SA}$, but not for Group Rd.

\section{Extinction}

The mean speeds for immediate and delayed extinction conditions are shown in Figure 1. An analysis of vari- 


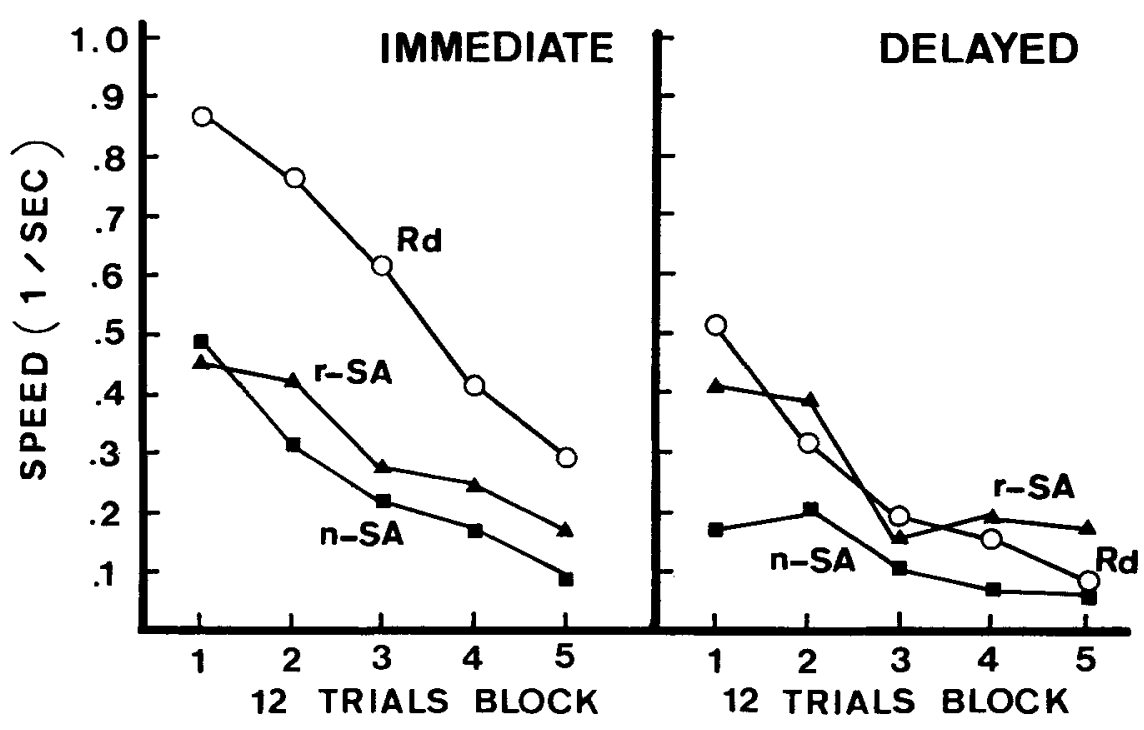

Figure 1. Mean running speed on each day of extinction for immediate and delayed extinction conditions. Experiment 1.

ance on the immediate extinction groups (7 rats in each group) indicated that there were significant groups $[F(2,18)=8.40, p<.01]$ and days $[F(4,72)=32.53$, $p<.01]$ effects, but that the groups $\times$ days interaction was not significant $(F<1)$. Tukey's posttests indicated that Group Rd ran significantly faster than the other two groups $(p s<.05)$, which did not differ from each other. In delayed extinction (seven rats in each group), there were significant groups $[F(2,18)=5.53, p<.05]$ and days $[F(4,72)=19.11, p<.01]$ effects. The interaction of two factors was also significant $[F(8,72)=3.50$, $p<.01]$. Tukey's posttests indicated that Group n-SA was less resistant than the other two groups $(p<.05)$, which did not differ from each other. To assess the performance change within each schedule group, statistical tests were performed between the immediate and delayed extinction groups. The rats trained with the $R d$ schedule showed the retention deficit; that is, the groups effect was significant $[F(1,12)=14.48, p<.01]$. On the other hand, those given the r-SA schedule and those given the n-SA schedule did not show a significant decline in resistance to extinction $(F \mathrm{~s}<1)$.

Figure 2 shows speeds of the first trial of each extinction day for the immediate and delayed extinction condi-

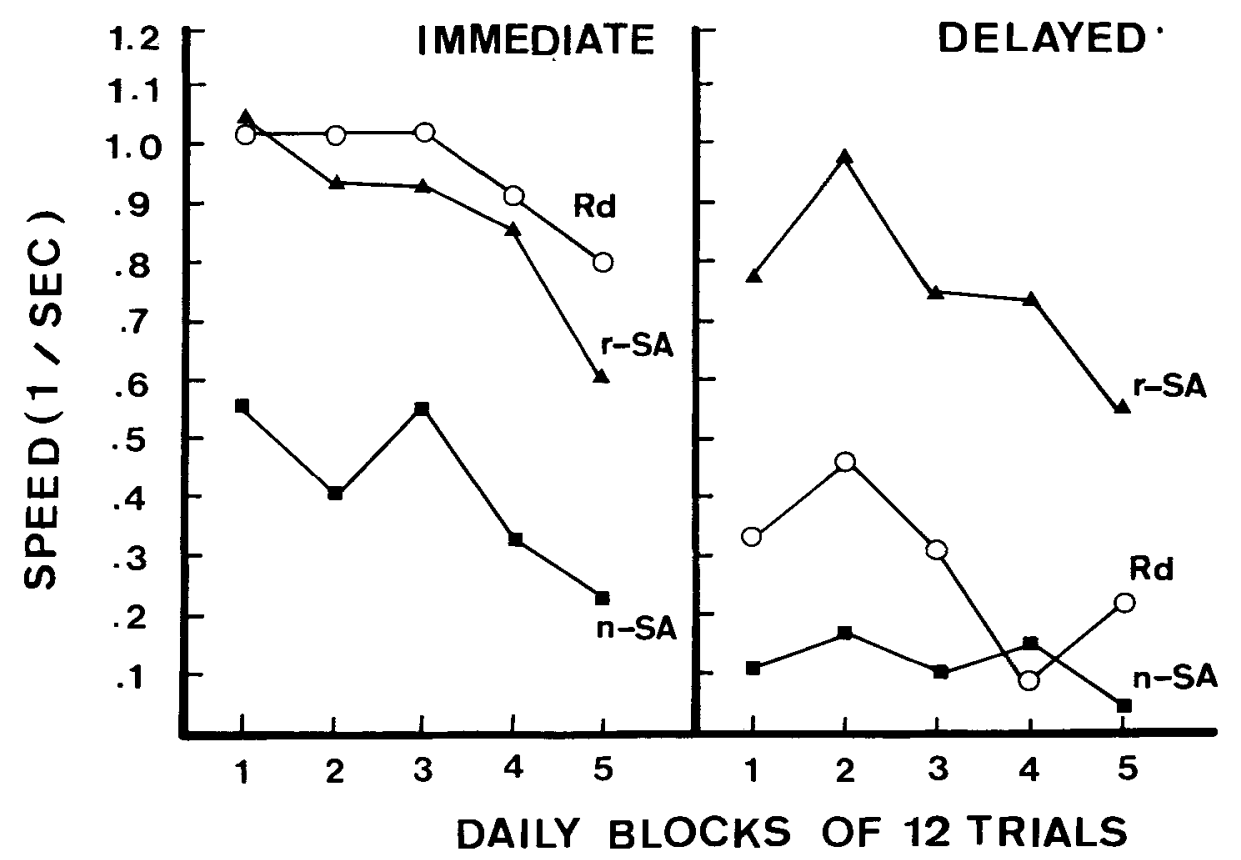

Figure 2. Speed of running on the first trial of each extinction day for immediate and delayed extinction conditions. Experiment 1. 
tions. Statistical tests similar to those done on the daily means were conducted. Groups Rd and r-SA, which did not differ from each other, responded more quickly than Group n-SA in immediate extinction. In delayed extinction, however, Group r-SA showed greater speed than the other two groups, which did not differ from each other. In a test of the speed change as a function of the A-E interval, Groups Rd and n-SA, unlike Group r-SA, showed a significant retention deficit.

At the terminal acquisition level, however, there were significant differences among threc basic groups, and this trend held true when each basic group was further divided into two groups. To assess extinction performance, the speed scores were transformed into rate measures, as recommended by Anderson (1963). The transformation corrected for different speeds at the end of acquisition. The mean speed on all trials on the last 2 days of acquisition was used to estimate the asymptote of acquisition, and the extinction asymptote was taken to be the reciprocal of $60 \mathrm{sec}(.016)$. Each extinction score was corrected with the equation: rate corrected speed $=$ (extinction trial - asymptote of extinction)/(asymptote of acquisition asymptote of extinction). Individual rats' transformed rates were averaged within group and are plotted in Figure 3.

In immediate extinction, an analysis of variance on the rate measure indicated that the groups $[F(2,18)=4.99$, $p<.05]$ and days $[F(4,72)=21.50, p<.01]$ effects were significant, as was the interaction of both factors $[F(8,72)=2.11, p<.05]$. Tukey's posttests revealed that Groups Rd and r-SA differed from each other to a negligible degree and showed greater resistance to extinction than Group n-SA. Similar tests performed on the delayed extinction groups indicated that both the groups effect $[F(2,18)=3.98, p<.05]$ and the days effect
$[F(4,72)=22.80, p<.01]$ were significant. The interaction of these two factors was also significant $[F(8,72)$ $=3.38, p<.1]$. Tukey's posttests indicated that Group r-SA was significantly more resistant than the other two groups, which did not differ from each other. Again, statistical comparisons were conducted on the rate measure for the immediate and delayed extinction conditions within each schedule group. Although the retention deficit was significant in the rats given the $\mathrm{Rd}$ schedule, those given the r-SA schedule and the n-SA schedule did not show a significant decline in resistance to extinction.

\section{Discussion}

The patterning effect in acquisition was demonstrated in both the r-SA schedule and the n-SA schedule. Our previous study (Ishida, 1981) failed to find the effect under reward conditions that were almost the same as those used in Experiment 1, except for the number of trials and the length of the intertrial interval (ITI). Thus, with the SA schedule, the extensive number of trials and the fairly short ITI may be important factors for the patterning effect. The effects on extinction will be discussed later.

The extinction results were complicated in part because of the terminal acquisition differences. In brief, (1) the rats given the r-SA schedule demonstrated considerable resistance to extinction in both immediate and delayed extinction, (2) the rats given the Rd schedule showed a decline in resistance to extinction over the long retention interval, and (3) those given the n-SA schedule demonstrated relatively low resistance in both extinction conditions, although the retention deficit was not greater than in the case of the Rd schedule.

Under immediate extinction, resistance to extinction among the three groups can, on the whole, be explained by the sequential variables, although Group $\mathrm{Rd}$ failed to

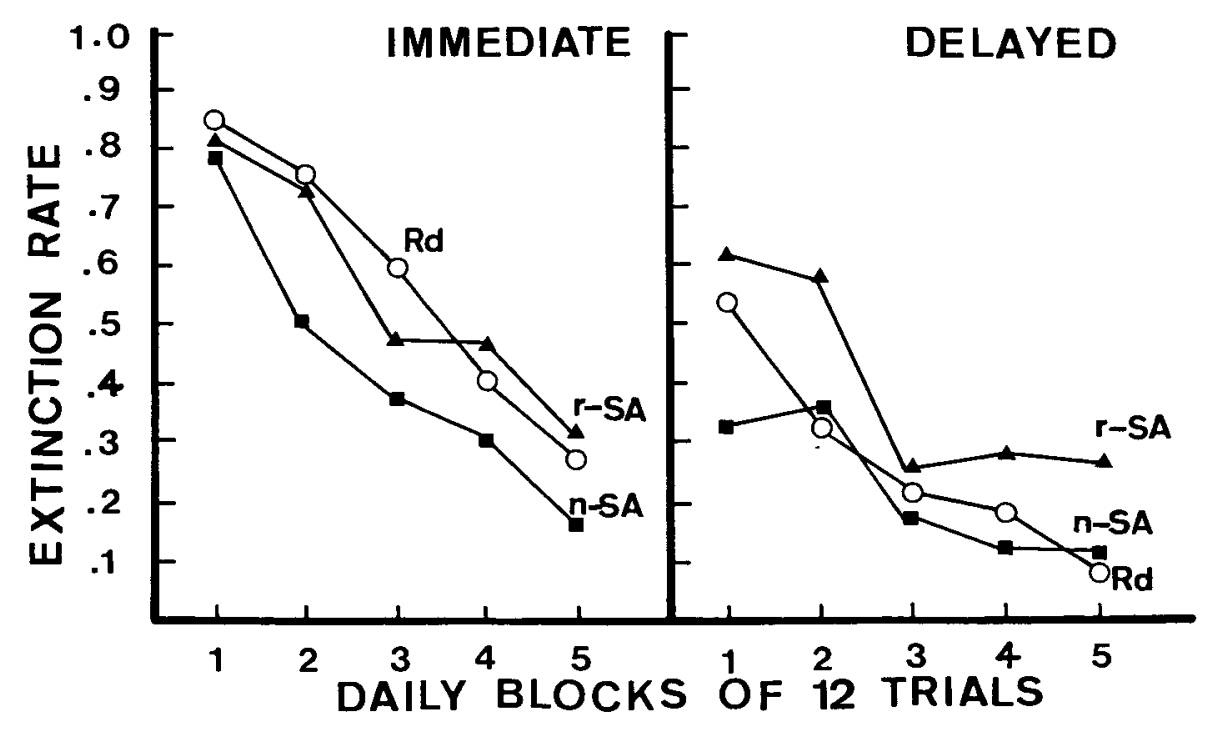

Figure 3. Rate-transformed resistance to extinction for immediate and delayed extinction conditions. Experiment 1. 
differ from Group r-SA on the basis of the rate measure. The sequential mechanisms (Capaldi, 1967, 1971) predict greater resistance to extinction in Group Rd than in the other two groups, since the former group includes more kinds of different $N$-lengths than the latter two groups. On the other hand, resistance to extinction under delayed extinction and the differential retention deficits among schedules cannot be explained by the $N$-length variable just mentioned.

However, resistance to extinction among groups is closely related to speed on the first trial of each extinction day. Especially the magnitude of resistance obtained under delayed extinction seems to depend on the type of trial on $T_{1}$ in acquisition. We cannot determine whether the important factor in regulating resistance to extinction is trial type on $T_{1}$ in acquisition or distribution pattern of reinforcement. Therefore the relative weights of effects of both factors were assessed separately in Experiment 2 .

\section{EXPERIMENT 2}

The results obtained in Experiment 1 on the whole confirmed those of the previous studies, which had reported that rats given a regularly alternating schedule showed persistent performance across long A-E intervals. On the other hand, Experiment 1 suggested that the effect of reward type of the first trial in acquisition was particularly important.

In Experiment 2, three basic groups were formed to clarify the relative effects of a reinforcement schedule and an $\mathrm{R}$ trial on $T_{1}$ during acquisition. For Groups $\mathrm{r}-\mathrm{Rd}$ and $r-S A$, the daily reinforcement sequence was always initiated by the $\mathbf{R}$ trial. For Group Rd, the reward sequence was randomized among trial types on $T_{1}$ and the remaining trials of each day. In addition, Groups $r-R d$ and $R d$ were equated in terms of the number of different $N$-lengths used ( $N_{1^{-}}, N_{2^{-}}$, and $N_{3}$-lengths were used in both groups). In acquisition, all groups of rats received an equal number of $\mathrm{R}$ and $\mathrm{N}$ trials.

If resistance to extinction under delayed extinction was exclusively regulated by the $\mathrm{R}$ trial on $T_{1}$, Group $\mathrm{r}-\mathrm{SA}$ would not be expected to differ from Group r-Rd, and these two groups would be more resistant than Group Rd. On the other hand, if resistance under delayed extinction was determined mainly by the alternating reward schedule, Group r-SA would be more resistant than the other two groups.

\section{Method}

Subjects

The subjects were 48 rats similar to those of Experiment 1. They were about 90 days old at the start of the experiment.

\section{Apparatus}

The apparatus was the same as that used in Experiment 1.

\section{Procedure}

Preliminary training, which lasted for 18 days, was identical to that used in Experiment 1 . At the end of this period, the rats were divided into three basic groups of 16 rats each ( $\mathrm{r}-\mathrm{SA}, \mathrm{r}-\mathrm{Rd}$, and Rd). Acquisition training was begun on Day 19. The rats received eight trials a day for 20 days (a total of 160 trials). At the end of acquisition training, each basic group was further divided into two groups. For half of the rats, extinction training was begun on the day after the end of acquisition (immediate extinction); for the other half, extinction training was begun on the 23 rd day after the end of acquisition (delayed extinction). All groups of rats received extinction training for eight trials a day for 8 days. The other procedures were the same as those used in Experiment 1.

\section{Results}

Four rats were removed during the experiment because of death and illness. A total of $\mathbf{4 4}$ rats were available for the analysis.

\section{Acquisition}

There were 14 subjects in Group r-SA and 15 in each of Groups Rd and r-Rd. An analysis of variance on the mean speeds for the three basic groups indicated that both the days effect $[F(19,779)=132.24, p<.01]$ and the groups $\times$ days interaction $[F(38,779)=1.50, p<.05]$ were significant, but that the groups effect was not significant $(F<1)$. There was no significant group difference on the last day of acquisition $(F<1)$. The means of the $\mathrm{R}$ trials and $\mathrm{N}$ trials on the last day of acquisition were, respectively, 0.76 and 0.65 for Group r-SA, 0.76 and 0.74 for Group r-Rd, and 0.81 and 0.85 for Group Rd. A $3 \times 2$ factorial analysis of variance revealed that only the interaction of groups $\times$ type of trial was significant $[F(2,41)=4.56, p<.05]$. Tukey's posttests indicated that Group r-SA ran rapidly on $\mathrm{R}$ trials and slowly on $\mathrm{N}$ trials, but that the other two basic groups did not run differentially.

\section{Extinction}

The mean speeds for immediate and delayed extinction conditions are shown in Figure 4. Twenty-one rats (7 in each group) were run under immediate extinction and 23 (7 in Group r-SA and 8 each in Groups Rd and r-Rd) were run under delayed extinction. In both extinction conditions, there was no significant difference among groups in the terminal level of acquisition.

Analysis of variance for the three immediate-extinction groups indicated that there were significant groups $[F(2,18)=4.57, p<.05]$ and days $[F(7,126)=38.62$, $p<.01]$ effects, but that the interaction of the two factors was not significant $(F<1)$. Tukey's posttests indicated that Group r-SA was significantly less resistant than the other two groups, which did not differ from each other. In delayed extinction, there were significant groups $[F(2,20)=14.41, p<.01]$ and days $[F(7,140)=23.44$, $p<.01]$ effects. The interaction was also significant $[F(14,140)=3.90, p<.01]$. Tukey's posttests revealed that the three groups differed significantly from each other. To assess the performance change as a function of the A-E interval, groups of same schedules in immediate and delayed extinction conditions were compared. A significant retention deficit was found in both the r-Rd 


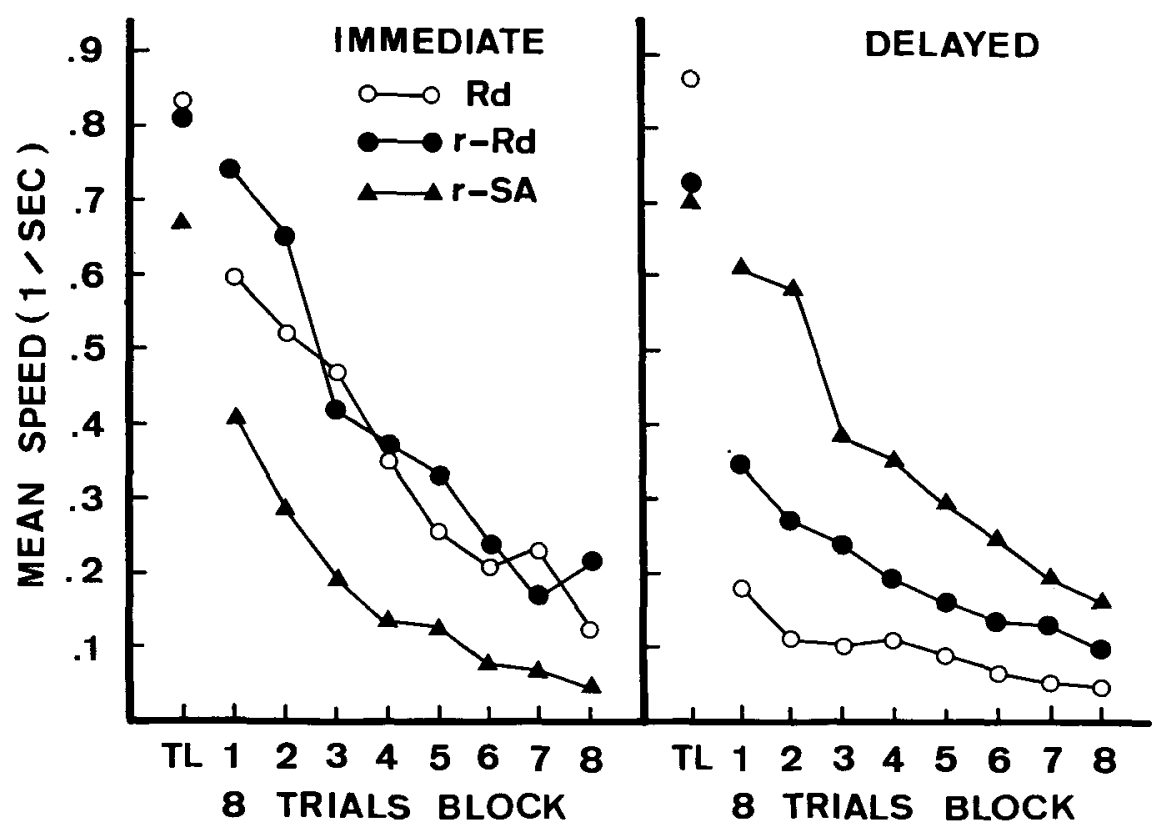

Figure 4. Mean running speed on each day of extinction for immediate and delayed extinction conditions. Experiment 2.

schedule and the $\mathrm{Rd}$ schedule $[F(1,13)=4.78$, and 8.78 , respectively, $p s<.05$ or better]. However, the rats given the r-SA schedule showed increased resistance to extinction, rather than a retention deficit $[F(1,12)=6.64$, $p<.05]$.

Figure 5 shows the speeds on the first trial $\left(T_{1}\right)$ of each extinction day and the means of the other seven trials $\left(T_{2-8}\right)$ for the immediate and delayed extinction conditions. In immediate extinction, there was no significant difference among groups on $T_{1}$ speed $(F<1)$, and the group difference on the mean of $T_{2-8}$ did not reach the acceptance level of significance $[F(2,18)=2.65, p<.10]$. In delayed extinction, the three groups differed significantly on $T_{1}$ speed $[F(2,20)=14.65, p<.01]$ and on the mean of $T_{2-8}[F(2,20)=9.32, p<.01]$. Again, groups of the same schedule in the immediate and delayed extinction conditions were compared. For the rats given the r-SA schedule, peformance increased significantly over a long retention interval both on $T_{1}[F(1,12)=8.94, p<.01]$ and on $T_{2-8}[F(1,12)=4.85, p<.05]$. Contrary to the case with the r-SA schedule, the retention deficit in rats given the Rd schedule was significant on both measures $\left[F(1,13)=4.94\right.$ and 10.61 on $T_{1}$ and $T_{2-8}$, respectively, $p s<.05$ or better]. However, in rats given the r-Rd schedule, the retention deficit was significant on $T_{2-8}$ $[F(1,13)=4.78, p<.05]$, but not on $T_{1}(F<1)$.

\section{Discussion}

The major findings of Experiment 2 were that (1) Group r-SA was more resistant than Group r-Rd under delayed extinction, and (2) the rats given the r-SA schedule showed no retention deficit over a long A-E interval, unlike those given both the r-Rd and Rd sched- ules. The former results indicate that a regularly alternating reward pattern is a more important factor than reward type on $T_{1}$ in determining the magnitude of resistance in delayed extinction. But the $\mathrm{R}$ trial on $T_{1}$ seems to raise performance level independently of the reward sequence, judging from the superior resistance of Group r-Rd over that of Group Rd in delayed extinction.

In immediate extinction, two groups (r-Rd and Rd), in which the values of $N$-length variables were equated as much as possible, failed to differ from each other. Both groups were more resistant than Group r-SA, in which only the $N_{1}$-length occurred repeatedly in its sequences. These results, under immediate extinction, are consistent with the predictions of Capaldi's $(1967,1971)$ sequential hypothesis. However, resistance to extinction under delayed extinction cannot be described by the $N$-length variables.

In Experiment 2, the PRDE was found, in which the superior resistance to extinction of the random schedule groups ( $R d$ and $r-R d)$ relative to the alternating schedule group ( $r-\mathrm{SA}$ ) disappeared when extinction was delayed. As has been shown in previous studies (Ishida, 1981, 1983), the PRDE is attributable to persistent performance despite a long retention interval in the alternating schedule group.

\section{GENERAL DISCUSSION}

The present two experiments were designed to assess the effect of reinforcement/nonreinforcement on $T_{1}$ on the PRDE. The results, especially those from Experiment 2, indicate that the occurrence of PRDE is regulated mainly by the regularly alternating reward pattern, and that the 


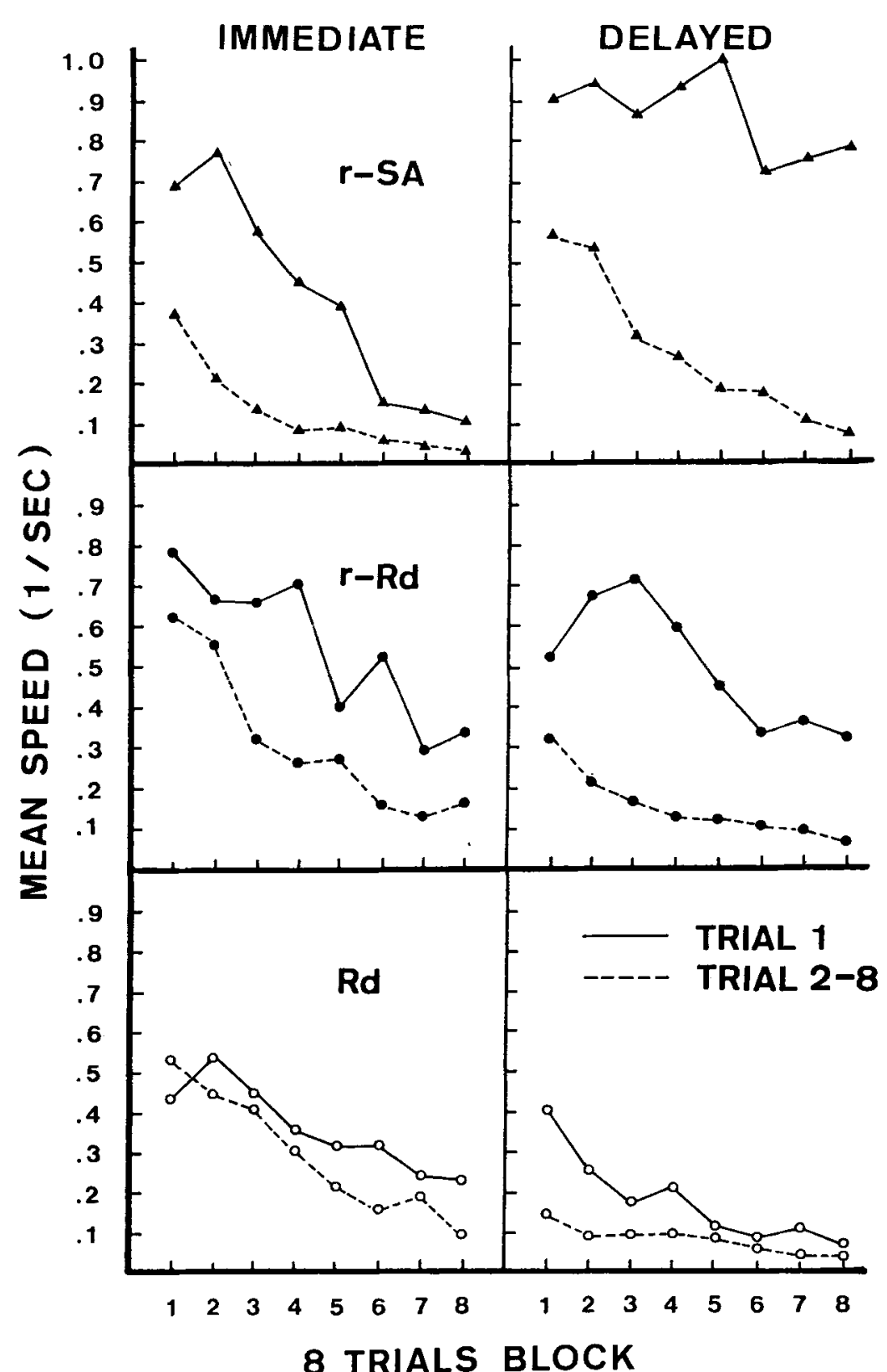

Figure 5. Speed on Trial 1 and the mean on the remaining seven trials (Trials 2-8) on each day of extinction for immediate and delayed extinction conditions. Experiment 2.

type of reinforcement on $T_{1}$ is not a determinant of the PRDE, although a reinforcement trial on $T_{1}$ encouraged resistance to extinction, as shown by the fact that Group r-Rd's resistance was superior to that of Group Rd under delayed extinction in Experiment 2. Specifically, reinforcement on $T_{1}$ seems to facilitate delayed extinction performance when this is accompanied by a regularly alternating reward pattern, as demonstrated in Experiment 2 when the rats given the r-SA schedule showed increased resistance to extinction over a long A-E interval, rather than a retention deficit. However, a fixed pattern of rein- forcement could play a more important role for persistent resistance to extinction, as suggested by the fact that even the rats given the n-SA schedule in Experiment 1 showed less of a retention deficit than those given a random schedule. More recently, Ishida (1984) found the PRDE using a regular $33 \%$ schedule, in which a NNR sequence occurred repeatedly (namely, first trial not rewarded). This schedule group showed no retention deficit.

The patterning effect in acquisition was found in the present two experiments, although it was not found in the previous studies, which used single-alternation (Ishida, 
1981) and regular 33\% (Ishida, 1984) schedules. A clear demonstration of this effect may not be a prerequisite condition for the PRDE and/or persistent resistance to extinction over a long retention interval.

The sequential mechanisms discussed by Capaldi (1967, 1971) can explain some aspects of the present results. A complete explanation of the results, including the PRDE phenomenon, will need to assume other mechanisms. One possible assumption is that the rats given an alternating schedule learn the regularity or rule of the reinforcement sequence, in parallel with which they acquire habit strength on each component (i.e., each value of $N$-length) through associative mechanisms. According to Hulse's rule-encoding models $(1978,1980$; see also Hulse \& Dorsky, 1977, 1979), rats, like humans, learn a pattern's formal structure, as well as the elements of a pattern. Hulse and his associates assumed that the rats formed an internal representation of a pattern's rule structure and that they used that representation as a mediator in recalling the pattern. Their assumption can be broadened as follows. The internal memory may act as a retrieval cue in recalling the elements during extinction.

However, the experimental data are not sufficient to conclude that internal memory is especially available in the delayed extinction situation. Perhaps the associative process (sequential mechanisms) and the cognitive process (rule-encoding mechanisms) have differential effects at different retention intervals. Making clear the relationship between the two processes might provide a complete explanation of the present results, including PRDE.

\section{REFERENCES}

Anderson, N. H. (1963). Comparison of different populations: Resistance to extinction and transfer. Psychological Review, 70, 162-179.

Bloom, J. M., \& CAPALDI, E. J. (1961). The behavior of rats in relation to complex patterns of partial reinforcement. Journal of Comparative \& Physiological Psychology, 54, 261-265.

Campbell, P. E., Knouse, S. B., \& Wroten, J. D. (1970). Resistance to extinction in the rat following regular and irregular schedules of partial reward. Journal of Comparative \& Physiological Psychology, 72, 210-215.

Capaldi, E. J. (1958). The effect of different amounts of training on the resistance to extinction of different patterns of partially reinforced responses. Journal of Comparative \& Physiological Psychology, 51, 367-371.

CAPALDI, E. J. (1967). A sequential hypothesis of instrumental learning. In K. W. Spence \& J. T. Spence (Eds.), The psychology of learning and motivation (Vol. 1). New York: Academic Press

CAPALDI, E. J. (1971). Memory and learning: A sequential viewpoint. In W. K. Honig \& P. H. R. James (Eds.), Animal memory. New York: Academic Press.

Capaldi, E. J., \& Minkoff, R. (1967). Reward schedule effects at a relatively long intertrial interval. Psychonomic Science, 9, 169-170.

HABU, Y., \& ONO, S. (1969). An information-theoretic approach to partial reinforzement effect: Determination of the effective order of entropy associated with reinforcement pattern. Japanese Psychological Research, 11, 76-87.

Hulse, S. H. (1978). Cognitive structure and serial pattern learning by rats. In S. H. Hulse, H. Fowler, \& W. K. Honig (Eds.), Cognitive processes in animal behavior. Hillsdale, NJ: Erlbaum.

HulSE, S. H. (1980). The case of the missing rule: Memory for reward vs. formal structure in serial-pattern learning by rats. Animal Learning \& Behavior, 8, 689-690.

HulsE, S. H., \& DoRSKy, N. P. (1977). Structural complexity as a determinant of serial pattern learning. Learning \& Motivation, 8 488-506.

HuLSE, S. H., \& DoRSKy, N. P. (1979). Serial pattern learning by rats: Transfer of a formally defined stimulus relationship and the significance of nonreinforcement. Animal Learning \& Behavior, 7, 211-220.

IsHIDA, M. (1981). Persistent resistance to extinction of single alternating reinforcement after long acquisition-extinction interval. Journal of General Psychology, 104, 191-201.

IsHIDA, M. (1983). Pattern memory and resistance to extinction in double alternating reinforcement. Journal of General Psychology, 109, 3-14.

IsHIDA, M. (1984). [Partial reinforcement delayed extinction effect under regular 33\% schedule] (p. 350). In Proceedings of 48th Annual Convention of Japanese Psychological Association. (In Japanese)

Jobe, J. B., Mellgren, R. L., Feinberg, R. A., Littlejohn, R. L., \& RIGBY, R. L. (1977). Patterning, partial reinforcement, and N-length effects at spaced as a function of reinstatement of retrieval cues. Learning \& Motivation, 8, 77-97.

Tyler, D. W., Wortz, E. C., \& Bitterman, M. E. (1953). The effect of random and alternating reinforcement on resistance to extinction in the rat. American Journal of Psychology, 66, 57-65.

(Manuscript received July 5, 1985; revision accepted for publication May 30, 1986.) 